\title{
Editorial
}

\section{Advanced Nonlinear Dynamics of Population Biology and Epidemiology}

\author{
Weiming Wang, ${ }^{1}$ Yun Kang, ${ }^{2}$ Malay Banerjee, ${ }^{3}$ and Kaifa Wang ${ }^{4}$ \\ ${ }^{1}$ College of Mathematics and Information Science, Wenzhou University, Wenzhou 325035, China \\ ${ }^{2}$ Science and Mathematics Faculty, School of Letters and Sciences, Arizona State University, Mesa, AZ 85212, USA \\ ${ }^{3}$ Department of Mathematics and Statistics, Indian Institute of Technology Kanpur, Kanpur 208016, India \\ ${ }^{4}$ School of Biomedical Engineering, Third Military Medical University, Chongqing 400038, China
}

Correspondence should be addressed to Weiming Wang; weimingwang2003@163.com

Received 6 November 2014; Accepted 6 November 2014; Published 22 December 2014

Copyright (C) 2014 Weiming Wang et al. This is an open access article distributed under the Creative Commons Attribution License, which permits unrestricted use, distribution, and reproduction in any medium, provided the original work is properly cited.

Modern biology and epidemiology have become more and more driven by the need of mathematical models and theory to elucidate general phenomena arising from the complexity of interactions on the numerous spatial, temporal, and hierarchical scales at which biological systems operate and diseases spread. Epidemic modeling and study of disease spread such as gonorrhea, HIV/AIDS, BSE, foot and mouth disease, measles, and rubella have had an impact on public health policy around the world which includes the United Kingdom, The Netherlands, Canada, and the United States. A wide variety of modeling approaches are involved in building up suitable models. Ordinary differential equation models, partial differential equation models, delay differential equation models, stochastic differential equation models, difference equation models, and nonautonomous models are examples of modeling approaches that are useful and capable of providing applicable strategies for the coexistence and conservation of endangered species, to prevent the overexploitation of natural resources, to control disease's outbreak, and to make optimal dosing polices for the drug administration, and so forth.

This special issue is concerned with the nonlinear dynamic modeling and related analysis of interacting populations and important epidemic diseases. All papers submitted to this special issue went through a thorough peer-refereeing process. Based on the reviewer's reports, we collect 50 original research articles by more than 100 active international researchers on differential equations. In the following, we briefly review each of the papers by highlighting the significance of the key contributions.
Twenty papers are concerned about the disease dynamics of differential equations on time scales. N. Wang et al. study the global stability of a multigroup SEIR epidemic model with general latency distribution and general incidence rate and define the basic reproduction number $R_{0}$ as the role of a threshold. L. Feng et al. present a mathematical model which combines the scale-free trait of Internet with the formation of P2P botnet and demonstrate that the model has a globally stable endemic equilibrium when the infection rate is greater than a critical value. C. Liu et al. establish a hybrid SIR vector disease model with incubation and show that there is a phenomenon of singularity inducing bifurcation as well as local stability switch around interior equilibrium when the economic interest increases forward zero. L. Qi et al. establish a mathematical model of schistosomiasis transmission under flood in Anhui province, China, and show that the diseasefree equilibrium is locally asymptotically stable if the basic reproduction number is less than one, and the stability of the unique endemic equilibrium may be changed under some conditions even if the basic reproduction number is larger than one. J. Wang et al. analyze the impact of seasonal activity of psyllid on the dynamics of Huanglongbing (HLB) infection and establish a new model about HLB transmission and show that if $R_{0}<1$, the disease-free periodic solution is globally asymptotically stable while if $R_{0}>1$ the disease persists. F. Wang et al. present an estimating formula for hospital potential capacity and demonstrate that the formula is useful to estimate the basic reproduction number in epidemiology. The results may contribute to the improvement of decision-making in the allocation of medical resources and 
the evaluation of the interventions and control efforts of the infectious disease. C. Yan and J. Jia study the local stability of the disease-free, endemic equilibria and Hopf bifurcation of a delayed SIR epidemic model with information variables and limited medical resources. Y. Pei et al. propose a delay SIR epidemic model with difference in immunity and successive vaccination and obtain that the basic reproduction number governs the dynamic behavior of the system. C. Huang and A. Fan study the relationship between antimicrobial resistance and the concentration of antibiotics with dynamical model of competitive population and indicate that long-term highstrength antibiotic treatment and prevention can induce the extinction of susceptible strain. M. Li and X. Liu investigate the disease dynamics of an SIR epidemic model with nonlinear incidence rate and show that the global properties of the system depend on both properties of these general functions. C. Dai et al. investigate the dynamic behavior of a viral infection model with general contact rate between susceptible host cells and free virus particles and give the local stability of the equilibria. M. A. Obaid and A. M. Elaiw propose and analyze two virus infection models with antibody immune response and chronically infected cells and give the global asymptotic stability of all steady states of the models. G. Li and G. Li consider an SIR endemic model in which the contact transmission function is related to the number of infected population and show that the model exhibits the bistability and undergoes saddle-node bifurcation, the Hopf bifurcation, and the Bogdanov-Takens bifurcation. H.-F. Huo and G.-M. Qiu study the dynamics of a malaria model and show that the disease-free equilibrium is globally asymptotically stable if $R_{0}<1$, and the system is uniformly persistent if $R_{0}>1$. C. Chen and Y. Xiao propose a mathematical model to consider the effects of saturated diagnosis and vaccination on HIV/AIDS infection and find that there exists a backward bifurcation which suggests that the disease cannot be eradicated even if the basic reproduction number is less than unity. When the basic reproduction number is greater than unity, the system is uniformly persistent. The findings suggest that increasing vaccination rate and vaccine efficacy and enhancing interventions like reducing share injectors can greatly reduce the transmission of HIV among IDUs in Yunnan province, China. J. Cui and Z. Wu consider an SIRS model incorporating a general nonlinear contact function and find that when the basic reproduction number $R_{0}<1$, the disease-free equilibrium is locally asymptotically stable, while when $R_{0}>1$, there is a unique endemic equilibrium that is locally asymptotically stable. P. Bi and H. Xiao consider a tumor-immune competition model with delay which consists of two-dimensional nonlinear differential equation and give the general formulas for the direction, period, and stability of the bifurcated periodic solutions are given for codimension-1 and codimension-2 bifurcations, including Hopf bifurcation and BT bifurcation. L. Wang et al. study a class of discrete SIRS epidemic models with nonlinear incidence rate and find that if basic reproduction number $R_{0}<1$, then the disease-free equilibrium is globally asymptotically stable, and if $R_{0}>1$, then the model has a unique endemic equilibrium and when some additional conditions hold, the endemic equilibrium is also globally asymptotically stable.
X. Zhou and X. Shi analyze a discrete-time-delay differential mathematical model that describes HIV infection of $\mathrm{CD} 4^{+} \mathrm{T}$ cells with drugs therapy and give the stability of the two equilibria and the existence of Hopf bifurcation at the positive equilibrium. K. Wang et al. propose a patch model for echinococcosis due to dogs migration and show that the dynamics of the model can be completely determined by $R_{0}$. If $R_{0}<1$, the disease-free equilibrium is globally asymptotically stable. When $R_{0}>1$, the model is permanent and endemic equilibrium is globally asymptotically stable.

Seven papers are developed to discuss the stochastic dynamics of population models. S. Zhao and M. Song consider the global existence and positivity of the solution and give sufficient conditions for the global stability in probability of a stochastic predator-prey system with BeddingtonDeAngelis functional response and stage structure. X. Ji and S. Yuan study the dynamics of a delayed stochastic model simulating wastewater treatment process and give the sufficient conditions for the stochastic stability of its positive equilibrium. F. Rao investigates an SIR epidemic model with stochastic perturbations and gives the existence of global positive solutions, stochastic boundedness, and permanence. L. Wang et al. study the stochastic dynamics of an SIRS epidemic model incorporating media coverage and find that if the intensity of noise is large, then the disease is prone to extinction, which can provide us with some useful control strategies to regulate disease dynamics. J. Zhao et al. investigate a stochastic SI epidemic model in the complex networks and show that the solution will oscillate around the disease-free equilibrium of deterministic system when $R_{0} \leq 1$, while it is persistent when $R_{0}>1$. F. Rao et al. investigate a Hassell-Varley type predator-prey model with stochastic perturbations and find some sufficient conditions for stochastically asymptotical boundedness, permanence, persistence in mean, and extinction of the solution. L. Zu et al. analyze the influence of stochastic perturbations on a singlespecies logistic model with the population's nonlinear diffusion among $n$ patches and give the sufficient conditions for stochastic permanence and persistence in mean, stationary distribution, and extinction.

Four papers focus on the traveling wave solutions. X. Tian and R. Xu investigate a delayed SIRS infectious disease model with nonlocal diffusion and nonlinear incidence and derive the existence of a traveling wave solution connecting the disease-free steady state and the endemic steady state. X. Wu et al. establish the existence of traveling wave solutions and small amplitude traveling wave train solutions of a reactiondiffusion system based on a predator-prey model incorporating a prey refuge and analyze the dynamic behavior of this model in the three-dimensional phase space. T. Zhang and Q. Gou consider the minimal wave speed of bacterial colony model with saturated functional response and give the existence and nonexistence of the traveling wave solutions. T. Zhang et al. investigate the spreading speed of a reactiondiffusion cholera model and find that there exists a traveling wave solution.

Four papers study the impulsive dynamics of population models. M. Zhao and C. Dai investigate the population 
dynamics of a three-species ecological system with impulsive effect and give the conditions for the system to be permanent when the number of predators released is less than some critical value. In particular, the authors find that less beneficial prey can support the predator alone when more beneficial prey goes extinct. J. Li considers a class of neural networks described by nonlinear impulsive neutral nonautonomous differential equations with delays and gives the criteria on global exponential stability. M. Zhao et al. investigate the dynamics of a Holling-Tanner predator-prey system with state-dependent impulsive effects and give the existence of periodic solution of the system with statedependent impulsive effects. Z. Luo investigates the existence of multiple positive periodic solutions of a class of impulsive functional differential equations with a parameter.

Five papers consider the stationary patterns in the reaction-diffusion equations. L. Zhang focuses on the pattern formation of a ratio-dependent food chain model and finds that the model dynamics exhibits complex pattern replication. X. Lian et al. investigate the spatiotemporal dynamics of a bacterial colony model and derive the conditions for Hopf and Turing bifurcations. L. Zhang and Z. Li focus on a spatially extended Holling-type IV predator-prey model that contains some important factors, such as noise (random fluctuations), external periodic forcing, and diffusion processes, and find that noise or external periodic forcing can induce instability and enhance the oscillation of the species density, and the cooperation between noise and external periodic forces inherent to the deterministic dynamics of periodically driven models gives rise to the appearance of a rich transport phenomenology. Y. Yuan et al. investigate the disease dynamics of a reaction-diffusion epidemic model and give the conditions of the existence and nonexistence of the positive nonconstant steady states, which guarantees the existence of the stationary patterns. Y. Wang et al. investigate a nonlinear reaction-advection-diffusion model of the interaction between nutrients and plankton and find that if the sinking velocity exceeds a certain critical value, the stable state becomes unstable and the wavelength of phytoplankton increases with the increase of sinking velocity.

Three papers investigate the predation dynamics. Y. Gao and S. Liu investigate a predator-prey model with dispersal for both predator and prey among $n$ patches and derive sufficient conditions under which the positive coexistence equilibrium of this model is unique and globally asymptotically stable if it exists. X. Feng et al. formulate and investigate a nonautonomous predator-prey model with infertility control in the prey and give the conditions for the permanence and extinction of fertility prey and infertility prey. X. Fan et al. study the global property in a delayed periodic predator-prey model with stage-structure in prey and density-independence in predator and give the sufficient conditions of the integrable form for the permanence and extinction.

There are seven new results in the special issue. Y. Peng and T. Zhang investigate stability and Hopf bifurcation analysis of a gene expression model with diffusion and time delay and give the local stability and delay-induced Hopf bifurcation. M. Li et al. establish the interaction model of two-cell populations following the concept of the randomwalk. After assuming that the cell movement is constrained by space limitation primarily, the authors analyze the model to obtain the behavior of two-cell populations as time is close to initial state and far into the future. P. Tang et al. establish a mathematical model for national fitness in China. The results indicate that national fitness put forward by the Chinese government is reasonable, and, in order to increase the number of people who frequently participate in sport exercise in a short period of time, if only one measure can be chosen, guiding people who never take part in physical exercise will be the best measure. X. Wang et al. presents a deterministic SATQ-type mathematical model for the spread of alcoholism with two control strategies and give some properties of the solutions to the model including positivity, existence, and stability. Y. Gao et al. give the sufficient conditions for the existence of at least two positive periodic solutions for a plant-hare model with toxin-determined functional response (nonmonotone). Y. Zhai et al. investigate the behavior of price differential equation model based on economic theory with two delays and show the linear stability and local Hopf bifurcation. W. Wang and B. Liu consider the shunting inhibitory cellular neural networks with time-varying delays in the leakage (or forgetting) terms and employ a novel argument to establish a criterion on the global exponential stability of pseudo-almost periodic solutions.

\section{Acknowledgments}

We would also like to thank the editorial board members of this journal, for their support and help throughout the preparation of this special issue.

Weiming Wang
Yun Kang
Malay Banerjee
Kaifa Wang




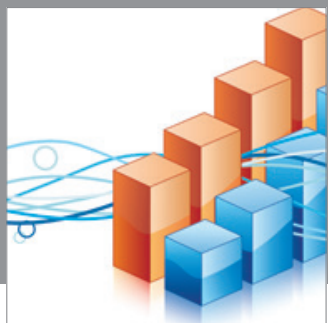

Advances in

Operations Research

mansans

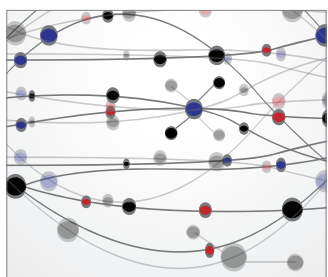

The Scientific World Journal
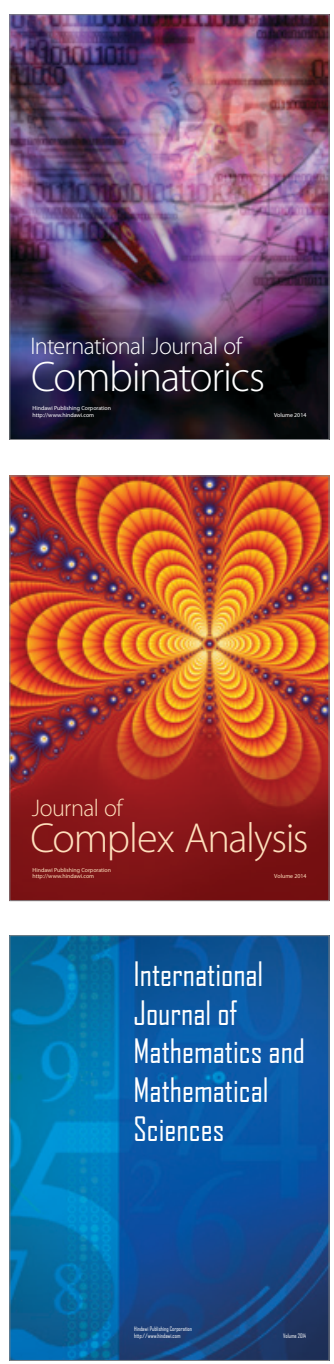
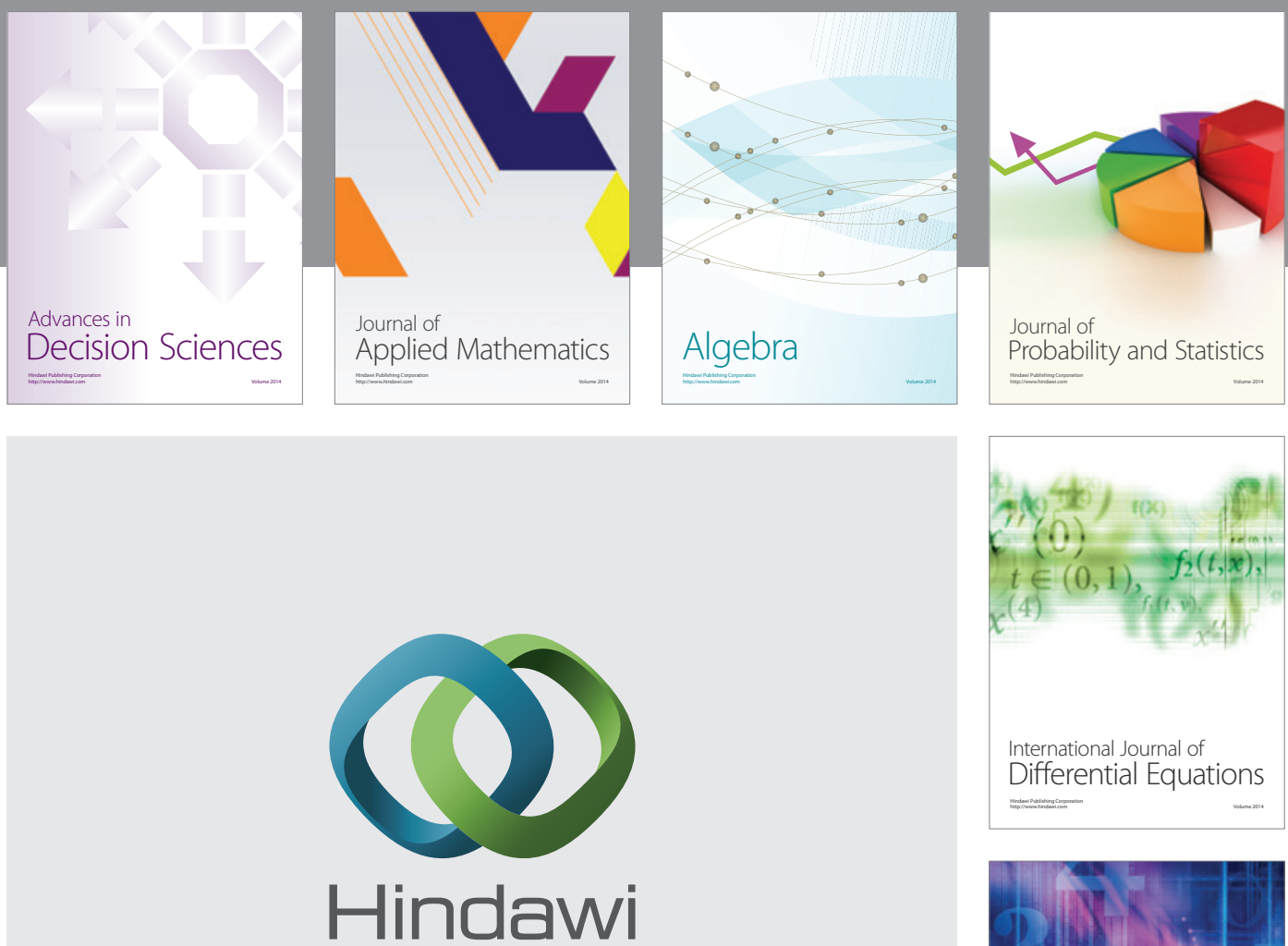

Submit your manuscripts at http://www.hindawi.com
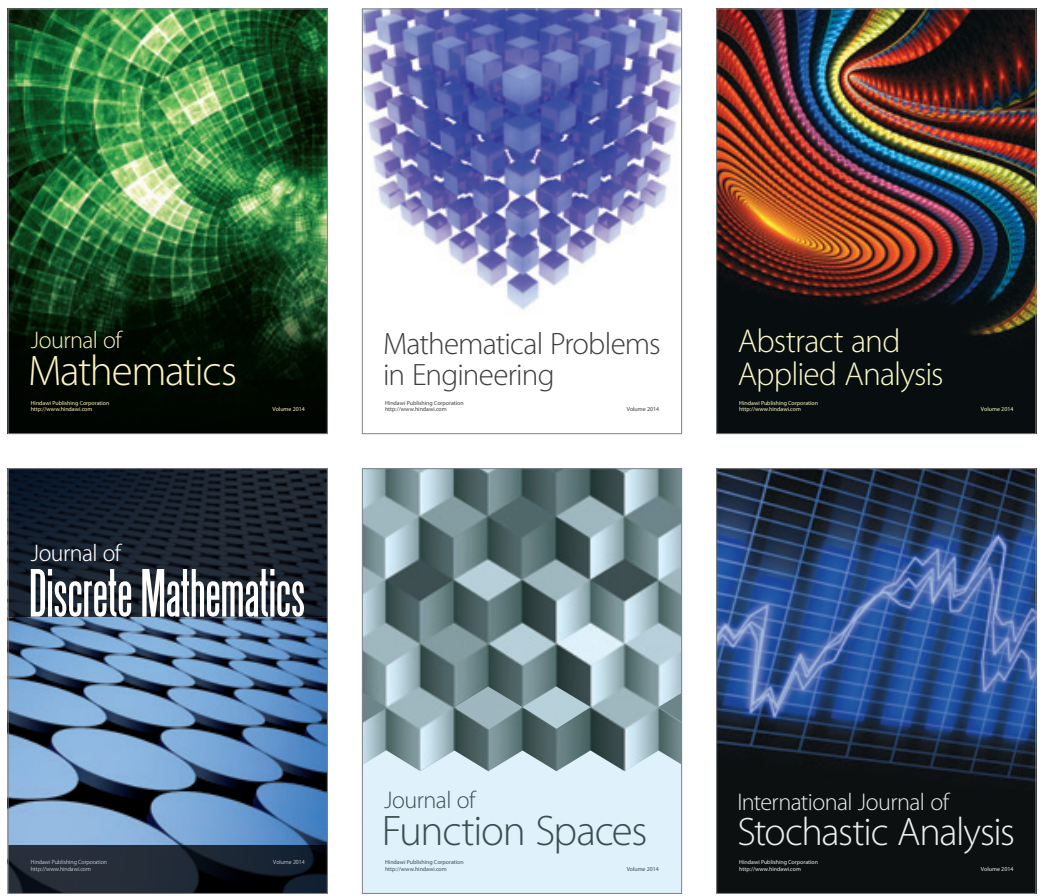

Journal of

Function Spaces

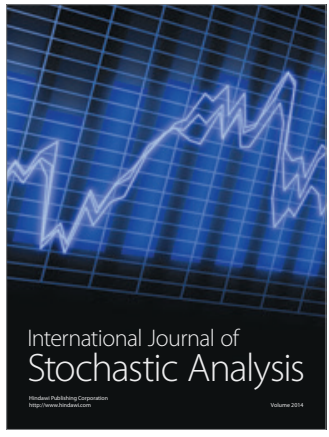

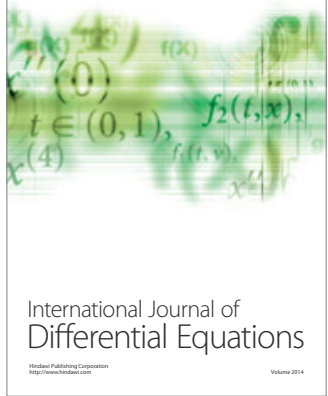
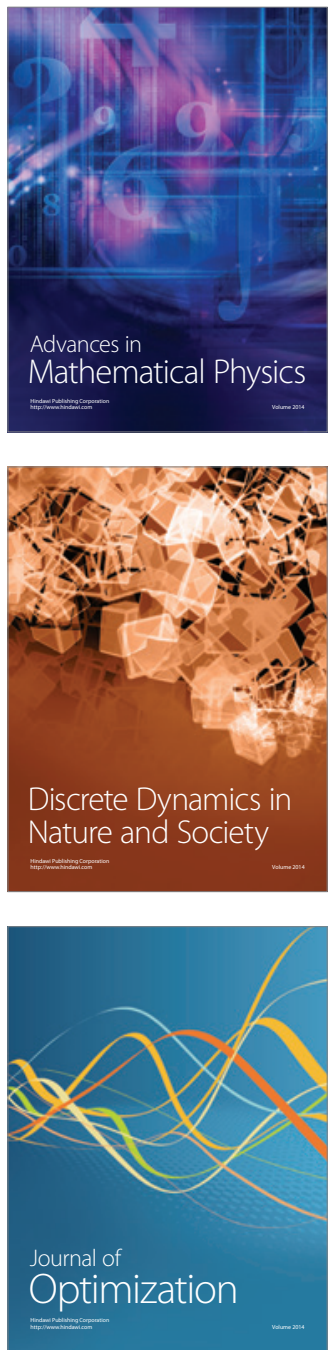